\title{
Hypoxic exercise as an effective nonpharmacological therapeutic intervention
}

\author{
Franck Brocherie (i) ${ }^{1}$ and Grégoire P. Millet (i] ${ }^{2}$
}

\section{Dear Editor,}

Lee et al. ${ }^{1}$ effectively reported how hypoxia signaling acts differently in organ systems, with protective hypoxiainducible factor (HIF) stabilization and downstream target pathway activation during acute hypoxic stress and HIF stabilization-related disease pathogenesis in chronic hypoxic conditions. The authors present different ways to target HIF pathways for organ protection, mainly through pharmacologic interventions (activators or inhibitors) or remote ischemic preconditioning/postconditioning (Table 2) ${ }^{1}$.

This review article, published in June 2019, suggests therapeutic applications of hypoxia that were also mentioned on 7 October, 2019, by the Nobel Assembly that delivered the 2019 Nobel Prize in Physiology or Medicine to William Kaelin Jr., Sir Peter Ratcliffe, and Gregg Semenza as follows: "Pharmacologically increased HIF function may aid in the treatment of a wide range of diseases, as HIF has been shown to be essential for phenomena as diverse as immune function, cartilage formation, and wound healing. Conversely, inhibition of HIF function could also have many applications: increased levels of HIF are seen in many cancers as well as in some cardiovascular diseases, including stroke, heart attack, and pulmonary hypertension". These researchers identified the transcription factor HIF-1 $\alpha$, which determines all oxygen-sensing responses, and clarified that it was regulated by Von Hippel-Lindau tumor suppressor depending on the oxygen level in cells. HIFs regulate more than 300 target genes (e.g., those for vascularization and angiogenesis, inflammation and immune function, autophagy and apoptosis, and redox homeostasis).

In this light, exercising in hypoxic conditions could also be a promising therapeutic approach for targeting HIF

\footnotetext{
Correspondence: Franck Brocherie (franck.brocherie@insep.fr)

'Laboratory Sport, Expertise and Performance (EA 7370), French Institute of Sport, 11, Avenue du Tremblay, 75012 Paris, France

${ }^{2}$ Institute of Sport Sciences, University of Lausanne, Lausanne, Switzerland
}

signaling in some chronic diseases ${ }^{2}$. Accordingly, there are a growing number of studies demonstrating the therapeutic benefits of combining exercise with hypoxia for blood pressure regulation ${ }^{3}$, chronic obstructive pulmonary disease ${ }^{4}$, obesity ${ }^{5}$, coronary artery disease ${ }^{6}$, or other pathologies. For instance, Gorgens et al. ${ }^{7}$ showed that combining muscle contraction and hypoxia resulted in HIF- $1 \alpha$ stabilization and improvements in glucose metabolism and insulin action in human skeletal muscle. With patients demanding to be exposed to safe hypoxia $(1800-3000 \mathrm{~m})$, the addition of exercise permits an increase in the overall hypoxia-induced metabolic stress (i.e., greater hypoxemia induced by muscle deoxygenation and systemic desaturation), resulting in putative physiological/therapeutic responses that are not activated to the same extent in a normoxic setting ${ }^{8,9}$. Evidence also suggests that adding high-intensity rather than moderateintensity exercise to hypoxic stimuli triggers greater adaptations (e.g., improvements in muscle oxygen homeostasis and tissue perfusion induced by enhanced mitochondrial efficiency, control of mitochondrial respiration, angiogenesis, and the capillary-to-fiber ratio $)^{10}$.

Overall, their findings opened doors for new methods based on any exercise modality that aims to reduce the oxygen supply and increase peripheral deoxygenation superimposed on hypoxia ${ }^{8,9}$. This approach may be relevant in the above-listed pathologies. Therefore, as part of the target and niches mentioned by Lee et al. ${ }^{1}$, exercising in hypoxia, particularly at high intensity, might be a valuable and viable nonpharmacological therapeutic strategy. Further investigation is warranted to identify optimal and individually tailored hypoxic exercise regimens and their synergistic effects with (accompanying) medication. 


\section{Conflict of interest}

The authors declare that they have no conflict of interest.

\section{Publisher's note}

Springer Nature remains neutral with regard to jurisdictional claims in published maps and institutional affiliations.

Received: 18 November 2019 Accepted: 17 January 2020.

Published online: 16 March 2020

\section{References}

1. Lee, J. W., Ko, J., Ju, C. \& Eltzschig, H. K. Hypoxia signaling in human diseases and therapeutic targets. Exp. Mol. Med. 51, 51-68 (2019).

2. Millet, G. P. et al. Therapeutic use of exercising in hypoxia: promises and limitations. Front. Physiol. 7, 224 (2016).

3. Serebrovskaya, T. V. et al. Intermittent hypoxia: cause of or therapy for systemic hypertension? Exp. Biol. Med. 233, 627-650 (2008).
4. Haider, T. et al. Interval hypoxic training improves autonomic cardiovascular and respiratory control in patients with mild chronic obstructive pulmonary disease. J. Hypertens. 27, 1648-1654 (2009).

5. Park, H. Y. et al. Exposure and exercise training in hypoxic conditions as a new obesity therapeutic modality: a mini review. J. Obes. Metab. Syndr. 27, 93-101 (2018).

6. Burtscher, M. et al. Intermittent hypoxia increases exercise tolerance in elderly men with and without coronary artery disease. Int J. Cardiol. 96, 247-254 (2004).

7. Gorgens, S. W. et al. Hypoxia in combination with muscle contraction improves insulin action and glucose metabolism in human skeletal muscle via the HIF-1alpha pathway. Diabetes 66, 2800-2807 (2017).

8. Faiss, R. et al. Significant molecular and systemic adaptations after repeated sprint training in hypoxia. PLOS ONE 8, e56522 (2013).

9. Brocherie, F. et al. Repeated maximal-intensity hypoxic exercise superimposed to hypoxic residence boosts skeletal muscle transcriptional responses in elite team-sport athletes. Acta Physiol. 222, e12851 (2018).

10. Hoppeler, H. \& Vogt, M. Hypoxia training for sea-level performance. Training high-living low. Adv. Exp. Med. Biol. 502, 61-73 (2001). 